\title{
Les effets sociaux de la transition en Hongrie
}

Les exemples du chômage, de la pauvreté et des migrations villes/ campagnes

Social impacts of the transition in Hungary. Examples of unemployment, poverty and urban/rural migrations

Einige soziale Konsequenzen der Transformation in Ungarn. Beispiele für Arbeitslosigkeit, Armut und territoriale Mobilität Stadt-Land

\section{Zoltán Dövényi}

\section{OpenEdition}

\section{Journals}

Édition électronique

URL : http://journals.openedition.org/rge/2467

DOI : 10.4000/rge.2467

ISSN : 2108-6478

\section{Éditeur}

Association des géographes de l'Est

\section{Édition imprimée}

Date de publication : 1 janvier 2003

ISSN : 0035-3213

Référence électronique

Zoltán Dövényi, « Les effets sociaux de la transition en Hongrie », Revue Géographique de l'Est [En ligne], vol. 43 / 1-2 | 2003, mis en ligne le 08 décembre 2010, consulté le 08 septembre 2020. URL : http:// journals.openedition.org/rge/2467; DOI : https://doi.org/10.4000/rge.2467

Ce document a été généré automatiquement le 8 septembre 2020

Tous droits réservés 


\section{Les effets sociaux de la transition en Hongrie}

Les exemples du chômage, de la pauvreté et des migrations villes/ campagnes

Social impacts of the transition in Hungary. Examples of unemployment, poverty and urban/rural migrations

Einige soziale Konsequenzen der Transformation in Ungarn. Beispiele für

Arbeitslosigkeit, Armut und territoriale Mobilität Stadt-Land

\section{Zoltán Dövényi}

\section{NOTE DE L'ÉDITEUR}

Cet article a été adapté et mis en forme par Julien Mathieu et Simon Edelblutte (CERPA / Université de Nancy 2)

\section{Introduction}

1 À la charnière des années 1980 et 1990, l'effondrement des régimes communistes des pays de l'Est a provoqué l'euphorie de populations convaincues que les changements politiques seraient suivis, plus ou moins rapidement, d'un essor économique et social. Mais, a posteriori et contrairement à l'espoir initial, la transition ne s'est pas effectuée en douceur et s'est accompagnée de graves problèmes économiques et sociaux. Le niveau de vie de la grande majorité des habitants des pays post-communistes européens est aujourd'hui plus bas qu'à la fin de la période communiste et un sentiment de nostalgie de l'ancien régime politique s'est donc développé dans de nombreuses couches de la population.

2 La Hongrie ne fait pas exception à cette règle, bien qu'elle ait été à l'époque le pays qui avait envisagé le post-communisme avec le plus de sérénité. Ainsi et après une 
décennie, nombreux sont les Hongrois qui s'interrogent sur les modalités de cette mutation promettant un avenir meilleur qui n'est toujours pas assuré aujourd'hui. D'après un sondage réalisé en 1995, 51 \% des Hongrois pensent que le nouveau régime est pire que l'ancien contre seulement $26 \%$ qui sont d'avis contraire (Ferge, 1996). Cette enquête fut cependant effectuée à l'époque où la situation économique du pays était à son plus bas niveau. Depuis, les indicateurs macroéconomiques se sont améliorées, mais au prix de lourdes restrictions touchant de larges portions de la population. Ainsi peut-on penser que ce sondage aurait reflété les mêmes réserves même après 1998, bien que l'économie hongroise ait connu depuis une reprise constante.

3 Face à cette situation, plusieurs questions peuvent être posées à juste titre. Quels sont les problèmes sociaux directement liés au changement de régime? Dans quelle mesure et pendant combien de temps sont-ils encore susceptibles d'accabler les Hongrois? Quand la population ressentira-t-elle enfin les effets positifs du changement économique? Cet article, réalisé dans le cadre du programme OTKA T16757, n'abordera que les principaux aspects de ces problèmes complexes en insistant sur le développement du chômage et de la pauvreté au sein de la population et du territoire hongrois.

\section{Le chômage, évolution globale}

4 La conséquence socio-économique la plus emblématique du changement de régime est sans nul doute le chômage. Pourtant, le tournant de 1988-89 est loin d'en être totalement à l'origine.

\section{A. Le chômage, un mal ancien renforcé par la transition}

5 Le chômage était effectivement plus ou moins latent sous le régime communiste et le discours du plein emploi développé par les communistes était un slogan et non une réalité. Si, dans un régime socialiste, le taux de chômage était estimé entre 1 et $3 \%$ de la population active (Ferge, 1988), bon nombre de personnes exerçaient en fait un emploi « inutile » - c'est le "chômage déguisé » des économistes. En Hongrie, de source officielle et avant 1990, on estimait que seulement $80 \%$ de la capacité de travail auraient été suffisants pour maintenir artificiellement le niveau de production (Fassmann, 1992). Des calculs antérieurs du gouvernement communiste hongrois qui préparait, en 1968, une réforme économique, avaient montré que le passage à une économie de marché aurait alors induit le chômage de près de 500000 personnes (Fóti, Illés, 1992). Cette information n'a évidemment pas été divulguée à l'époque, mais confirme l'ancienneté des problèmes de chômage dans le marché du travail hongrois.

Renforçant ce chômage latent, la crise économique liée au changement de régime s'est accompagnée d'une baisse spectaculaire du nombre d'emplois. Ainsi, pendant la première moitié des années quatre-vingt-dix, près de 1,4 million de postes ont-ils été supprimés, excédant le nombre des emplois créés pendant les quatre décennies précédentes (Tímár, 1997). De ce fait, le taux d'activité de la population a fortement diminué et, aujourd'hui, reste largement inférieur à celui des pays capitalistes développés.

7 Étroitement lié à la crise économique, le ratio entre actifs et inactifs a fondamentalement évolué, passant de 116 inactifs ou personnes à charge pour 100 actifs en 1990, à 182 cinq ans plus tard. De tels chiffres n'avaient été enregistrés en 
Hongrie qu'au début du siècle dernier (Tímár, 1997). Cette situation nuit évidemment aux systèmes de sécurité sociale et de retraite, rendant la croissance de la population active et la réduction du taux de chômage indispensables dans un avenir proche. La situation est d'autant plus grave que près des deux-tiers des 1,5 million de personnes ayant perdu leur emploi ont en réalité quitté le marché du travail, devenant inactifs et ne participant donc pas à la croissance du taux de chômage. Le solde a bien sûr alimenté la croissance d'un taux de chômage déjà massif et le développement de l'économie souterraine.

\section{B. Les phases du chômage depuis la transition}

En Hongrie, les chômeurs sont officiellement enregistrés et comptabilisés depuis le début de l'année 1989. Depuis, l'évolution du taux de chômage a connu trois périodes principales :

- une période de chômage rampant, depuis le début de l'année 1989 jusqu'à l'été 1990, se caractérise par un lent accroissement du taux de l'ordre de $1 \%$ pendant l'été 1990 . À cette époque, à la suite du passage à une économie de marché, la Hongrie était optimiste car il semblait que la transition économique pouvait s'effectuer sans induire de graves problèmes d'emplois. Mais ces espoirs ont été déçus...;

- une période de chômage « galopant » de l'été 1990 aux premiers mois de l'année 1993, est caractérisée par le développement massif du chômage. Le nombre de chômeurs est alors passé de 40-50 000 à 700000 avec un taux de croissance moyen compris entre 1 et $13 \%$ par an. Le pays prit alors conscience de l'ampleur du phénomène et des problèmes qu'il générait d'un point de vue économique et social ;

- une période de chômage dégressif se poursuit depuis le pic de février 1993, correspondant à une baisse continue du nombre de chômeurs : au mois de décembre 1997, il y avait 464000 chômeurs enregistrés, soit 10,4 \% de la population active ; ce pourcentage s'est abaissé à 5,6\% en 2002 (OCDE).

Ce taux place la Hongrie dans une position plutôt favorable, non seulement face aux autres pays candidats à l'Union Européenne (République Tchèque, 7,3\%; Pologne, $19,9 \%$ ), mais aussi par rapport à de nombreux membres actuels de l'Union (France, $8,8 \%$; Espagne, $11,3 \%$ ).

10 Les conséquences les plus dramatiques de l'adoption du nouveau système économique ont donc eu lieu entre 1990 et 1993. La crise économique s'est traduite non seulement par un accroissement exponentiel du taux de chômage, mais elle n'a pas touché les différentes catégories sociales hongroises et les différentes régions de la même façon.

\section{Les caractéristiques du chômage en Hongrie}

11 Le chômage hongrois n'est évidemment pas uniforme. Il concerne des régions et des groupes sociaux variés.

\section{A. Les caractéristiques structurelles}

12 Ainsi, il existe une différence sensible entre les taux de chômage masculin et féminin. La situation hongroise dans ce domaine est originale dans les pays d'Europe centrale, puisque le taux de chômage des femmes, à la suite du changement de régime, est 
devenu largement moins élevé que celui des hommes. La principale explication de cette inégalité devant l'emploi réside dans le fait que beaucoup d'emplois typiquement masculins - notamment industriels - ont disparu. Cependant, la mise en place progressive d'une économie de marché a amenuisé cette inégalité des sexes face au chômage, même si les femmes sont aujourd'hui toujours moins touchées que les hommes par le phénomène.

Le taux de chômage varie également en fonction du degré de formation. Lors de la transition entre les deux régimes, de très nombreux manœuvres, ouvriers spécialisés sans formation et employés de bureau ont, les premiers, perdu leurs emplois. Puis, le chômage a menacé des catégories socio-professionnelles mieux formées et, en 1997, les ouvriers qualifiés constituaient 36 à 37 \% des chômeurs. Par ailleurs, le chômage touche différemment les hommes et les femmes selon leur niveau de formation. En effet, le chômeur-type, en Hongrie, est un ouvrier qualifié, alors que les femmes de ce niveau de formation trouvent plus facilement du travail. À l'inverse, les femmes titulaires du baccalauréat sont plus touchées par le chômage que les hommes au diplôme équivalent. De même, entre diplômés de l'enseignement supérieur, le chômage féminin est plus important que celui des hommes (Lakatos, 1997).

14 En outre, en Hongrie, le chômage touche toutes les classes d'âge, ce qui, dans le contexte de la transition, est plutôt un aspect positif. Néanmoins, la proportion des chômeurs de moins de 30 ans a légèrement augmenté ces dernières années et dépasse en 1997 les $40 \%$, chiffre cependant inférieur - et de beaucoup - aux chiffres de nombreux pays européens voisins. Mieux encore, le changement de régime s'est accompagné de la croissance des étudiants du secondaire et du supérieur. Par exemple, les effectifs de l'enseignement supérieur ont doublé, améliorant d'autant plus la formation professionnelle et limitant le taux de chômage des moins de 30 ans.

La répartition par secteurs d'activité des chômeurs a également subi des modifications ces dernières années. Lors de la transition entre les deux régimes, la majorité des emplois supprimés concernaient l'industrie et l'agriculture. Parallèlement, le nombre d'employés du commerce, des transports et des services a augmenté, épongeant en parties les pertes des secteurs primaires et secondaires et développant donc le secteur tertiaire. La profonde transformation de l'industrie prit cependant fin en 1993-1994 et, depuis, le nombre d'emplois du secteur secondaire a augmenté, provoquant une baisse du taux de chômage des ouvriers.

16 L'aspect le plus grave concernant la situation de l'emploi en Hongrie est certainement le chômage de longue durée. En effet, la durée moyenne du chômage en Hongrie est longue, atteignant 240 à 250 jours et, près d'un quart des chômeurs de la fin des années 1990 avaient perdu leur emploi depuis plus de deux ans. Le chômage de longue durée affecte de très nombreuses personnes sans formation ou dont les qualifications sont peu recherchées et peu adaptées au marché du travail actuel; nombre d'entre-elles sont tziganes. Cette catégorie de population dépend donc de plus en plus de diverses aides sociales.

17 Un dernier facteur aggravant existe. Le chômage touche de plus en plus les actifs des familles nombreuses. En effet, durant l'été 1997, un ménage de 2 personnes sur vingt avait un membre au chômage alors que ce rapport s'élevait à un sur six pour les ménages de 5 personnes et plus. 


\section{B. Les caractéristiques spatiales} b) supérieur à la moyenne, connaissent en général un taux de chômage plus faible (fig. 1). C'est à Budapest que cette relation est la plus spectaculaire car le taux de chômage est deux fois moins élevé que la moyenne nationale alors que le $\mathrm{PNB} / \mathrm{hab}$ est au contraire deux fois plus élevé. Les comitats de la Transdanubie du nord présentent également de bons résultats : le chômage y est inférieur à la moyenne nationale pour un PNB/hab plus élevé. Par contre, au nord et au nord-est (comitats de Nógrád, BorsodAbaúj-Zemplén et Szabolcs-Szatmár-Bereg), un PNB/hab bas se conjugue avec les plus forts taux de chômage.

Figure 1 : Chômage et revenu par habitant, par comitat.

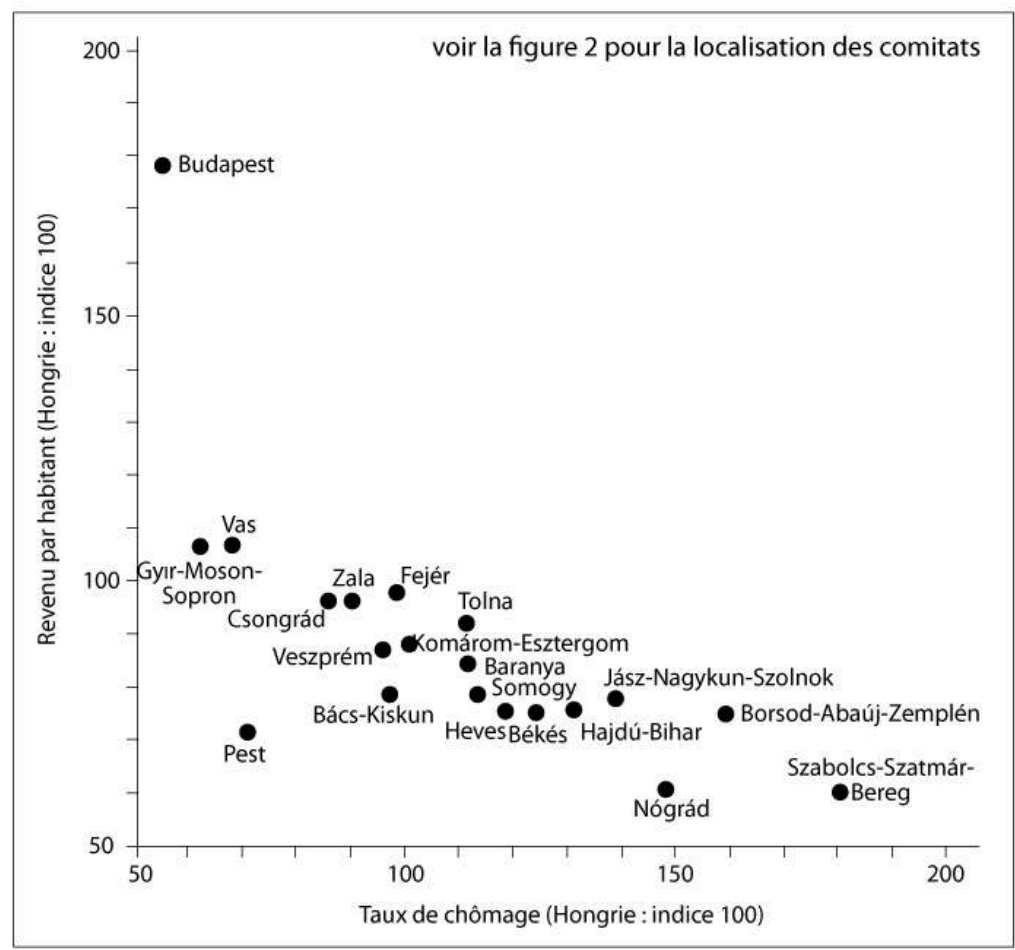

Source: Magyarország Statisztikai Evkınyve, réalisation :Z. Dövényi, informatique : S. Edelblutte

La carte du chômage au niveau des districts (fig. 2) permet d'affiner l'étude de ces inégalités régionales et de tenter de les expliquer; les variations géographiques enregistrées par le taux de chômage exprimant en effet la performance économique et la faculté d'adaptation d'un territoire à la crise économique. 




Ainsi, la carte révèle que les taux les plus faibles concernent de rares districts autour de Budapest et en Transdanubie du nord-ouest, le long de la frontière avec l'Autriche. Ces districts ont en effet de bons potentiels - notamment une situation favorable à proximité de l'Union Européenne (UE) - et sont prêts à accepter l'innovation et les capitaux. Le taux de chômage y reste donc toujours en-dessous de la moyenne nationale.

Par contre, de forts taux de chômage - largement supérieurs à la moyenne nationale se concentrent aux frontières du Nord-Est et de l'Est (Dövényi, 1995), qui, traditionnellement depuis un siècle, ont toujours été moins développées et moins riches.

L'Alföld (Grande Plaine hongroise) est également fortement affecté par un chômage élevé, principalement lié à deux facteurs. Le premier est la dissolution en $1991 \mathrm{du}$ Conseil d'Aide Économique Mutuelle $\left(\mathrm{CAEM}^{2}\right)$ qui a provoqué la perte de débouchés d'une agriculture locale aux structures déjà désorganisées par la transition économique. Le second est la pauvreté des investissements industriels effectués par le régime communiste dans cette région, ce qui a conduit à la faillite des usines obsolètes, alimentant un chômage déjà élevé, encore renforcé par la faible urbanisation de cette région.

Enfin, une dernière zone connaît d'importants taux de chômage. Ce sont les anciennes régions industrielles et minières actuellement en crise, notamment au nord-est du pays. Les industries lourdes et les mines, favorisées par l'État sous l'ère communiste, ne sont plus compétitives dans une économie de marché et périclitent rapidement depuis 1990.

Une dernière inégalité territoriale, entre les villes et les campagnes, existe dans le domaine du chômage. Pour l'opinion publique, le chômage est un phénomène essentiellement urbain, alors qu'en réalité, ce fléau est majoritairement rural en Hongrie. Les petits villages, traditionnellement peu développés, ont subi de plein-fouet la crise socio-économique, sans pouvoir développer d'activités relais à l'agriculture ou 
à la petite industrie rurale. Le développement du chômage risque, dans ces zones rurales profondes, de provoquer une véritable désintégration des sociétés rurales locales. C'est donc une menace pour les campagnes (Tolnai, Dövényi, 1995), d'autant plus que le développement du chômage s'accompagne évidemment d'une aggravation de la pauvreté.

\section{Pauvreté et migrations villes/campagnes}

Le changement de régime, en Hongrie, a également affecté le niveau de vie de la population, s'accompagnant tant de l'appauvrissement de la majorité de la population que de l'enrichissement d'une minorité. Bien évidemment, cela ne signifie pas que, sous le régime communiste, il n'existait pas de différence de revenus entre Hongrois, mais les personnes vivant sous le seuil de pauvreté étaient moins nombreuses qu'à l'heure actuelle.

\section{A. Le développement de la pauvreté}

Les statistiques fiables étant très rares en Hongrie dans ce domaine, seules des estimations concernant le nombre des pauvres avant et après le changement de régime seront utilisées. Dans la première moitié des années 1980, selon Andorka (1997), il y avait environ un million de pauvres en Hongrie. Bokor (1988) estime ce même chiffre à 1,5 million et d'autres avancent que 10 à $30 \%$ des Hongrois, c'est-à-dire de 1 à 3 millions de personnes, vivaient sous le seuil de pauvreté pour la même période (Ferge, 1986).

Ces estimations s'accordent donc assez bien et le nombre de pauvres était donc élevé sous le régime communiste. Cependant, les différences de revenus étaient beaucoup moins accusées qu'aujourd'hui.

Dans les années 1970 et 1980, le revenu des $10 \%$ des Hongrois les plus riches était quatre à cinq fois supérieur à celui de $10 \%$ des plus pauvres, ce qui correspondait alors à la situation des pays scandinaves ${ }^{3}$. Après le changement de régime, les différences de revenus se sont rapidement accusées. Le nombre des personnes vivant au-dessous du minimum vital dépassait les 2 millions. En 1996, on l'estime même entre 3,5 et 4 millions (Andorka, 1997). Parallèlement, les différences de revenus se sont accentuées. En 1996, le revenu de 10 \% des Hongrois plus riches était environ huit fois plus élevé que celui de $10 \%$ des plus pauvres. Comparé à la fin des années 1980, 1/5 seulement des Hongrois a vu ses revenus croître et les $4 / 5$ restant se trouvent dans une situation moins favorable qu'auparavant (fig. 3). 
Figure 3 : Répartition des revenus des ménages hongrois par déciles

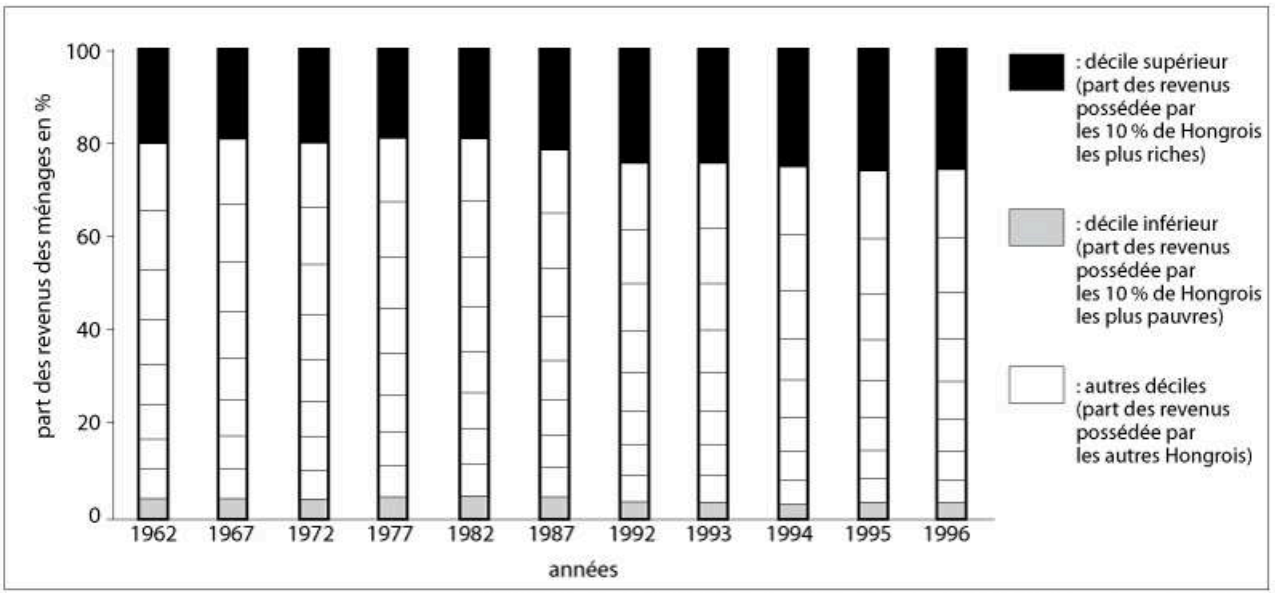

Source : Ehrlich, Révész, Tamási (1994), Andorka (1997), réalisation : Z. Dövényi, informatique : S. Edelblutte même récemment celui de la France ${ }^{4}$. Les bénéficiaires de cette mutation sont peu nombreux et sont estimés à environ un million de personnes qui vivent aisément. Par contre, une majorité de la population peut-être considérée comme perdante et la structure de la société se différencie de plus en plus de celle des États d'Europe de l'Ouest. À côté d'une élite très restreinte ayant des revenus élevés, la classe moyenne perd de plus en plus de terrain et glisse progressivement vers le bas de l'échelle sociale, alimentant une classe pauvre toujours plus importante (Andorka, 1997). La pauvreté est donc devenue un phénomène massif, c'est-à-dire qu'elle a pénétré des groupes sociaux qui n'avaient jamais connu pareille situation auparavant, tels que les chômeurs, les retraités, les grandes familles, les Tziganes... tous confrontés à de nouveaux problèmes de santé, d'alimentation, de logement...

D'un point de vue spatial, la pauvreté touche en premier lieu les campagnes déjà concernées par de forts taux de chômage (fig.2), même si ce dernier n'en est évidemment pas la seule cause. Elle n'est toutefois pas inconnue en milieu urbain, mais ses manifestations sont alors différentes de celles des campagnes. En effet, les pauvres se divisent, dans les villes, en deux groupes distincts : les retraités et les Tziganes ; ces derniers ayant tendance à se regrouper et donc à former de véritables ghettos.

\section{B. Les migrations villes/campagnes}

Avec la fin de l'ère communiste, un retournement complet des rapports démographiques villes/campagnes s'est produit en Hongrie. Auparavant, les principales migrations internes profitaient aux grandes villes, vidant les campagnes alentour dans un classique phénomène d'exode rural. L'exurbanisation bien connue des états d'Europe occidentale était donc inconnue en Hongrie jusqu'à la fin des années 1980.

Le changement de régime et le passage à l'économie libérale ont remis en cause ces mouvements. La population urbaine a en effet commencé à baisser et, dans l'histoire récente de la Hongrie, un tel phénomène n'avait jamais été observé, sauf lors des deux guerres mondiales. Cette nouvelle tendance traduit une mutation fondamentale: le changement de direction des migrations. Ainsi, l'exode rural observé sous le régime 
communiste a laissé la place à un mouvement inverse d'exurbanisation exponentielle vers les villages qui deviennent dès lors de plus en plus résidentiels.

Les données statistiques confirment cette évolution : entre 1990 et 1997, la population hongroise a diminué de $1,7 \%$ et la population urbaine de $3,7 \%$. Par contre, la population rurale a augmenté de $1,7 \%$. Cependant, cette croissance ne concerne essentiellement que les villages de plus de 500 habitants, alors que les plus petits villages continuent de décliner, parfois même jusqu'à la disparition...

Les villages ayant connu une hausse démographique sont évidemment ceux situés à proximité des grandes villes du pays, profitant donc de l'accélération des mouvements d'exurbanisation. Parmi les grandes villes, Budapest est sans conteste le meilleur exemple. Sa population a baissé de 130000 personnes entre 1990 et 1996, dont plus de $40 \%$ sont allés habiter en grande périphérie rurale et font donc partie du mouvement d'exurbanisation.

En Hongrie, les populations exurbanisées se divisent en deux groupes. Le premier est constitué des classes aisée et moyenne construisant ou achetant un pavillon dans la couronne périurbaine, alors que le deuxième est représenté par les pauvres ne pouvant plus vivre en ville pour des raisons financières et choisissant de migrer vers des villages moins attractifs et plus éloignés où la vie est moins chère (Dövényi, Kovács, Kok, 1997).

Pauvreté et migrations internes en Hongrie sont donc liées. La première alimentant constamment les secondes et en réorientant les flux, alimentant parfois encore plus le chômage et la pauvreté de régions rurales et peu développées déjà en difficulté.

\section{Conclusion}

Le chômage et la pauvreté sont donc deux des principales conséquences socioéconomiques de la transition de la Hongrie d'un régime communiste vers un régime libéral. Si la situation a aujourd'hui tendance à se stabiliser d'un point de vue macroéconomique, avec un taux de chômage tombant en dessous de 10 \%, les inégalités entre une élite restreinte très fortunée et une masse de pauvres se creusent de plus en plus. De même et d'un point de vue spatial, le gradient socio-économique entre la Hongrie occidentale et la Hongrie orientale s'accroît dangereusement. Plus riche et moins touchée par le chômage, bien intégrée déjà aux réseaux urbains et routiers de l'Europe occidentale, la Transdanubie du nord-ouest s'oppose aujourd'hui à une Hongrie orientale et méridionale frontalière d'états en grande difficulté économique et/ou politique (Ukraine, Roumanie, Serbie).

39 Ainsi, la déception de beaucoup de Hongrois est à la mesure de leurs attentes après le changement - pacifique et inespéré - en 1989. Ce sentiment alimente une certaine méfiance vis-à-vis de l'intégration portant décidée à une UE souvent présentée comme très libérale et faisant peu de cas des inégalités sociales. Il risque de provoquer une volonté de repli sur soi et des crispations nationalistes, voire nostalgiques de l'ancien régime. C'est pourquoi l'UE se doit de prendre en compte les inégalités socioéconomiques et spatiales croissantes dans tous les pays de l'Europe centrale et orientale, à un moment où ils redeviennent attractifs pour les Investissements Directs Étrangers (IDE) ${ }^{5}$. Le faible coût de leur main d'œuvre et le marché potentiel qu'ils constituent devrait favoriser ce rattrapage. 


\section{BIBLIOGRAPHIE}

Andorka R. (1997). - A Magyar társadalom változasai 1989-1996 (Les changements de la société hongroise entre 1989-1996), Ezredforduló, n² 2, p. 8-12.

Bokor Á. (1988). - Szegénység a 80-as évek elején közepen Magyarországon (Pauvreté en Hongrie dans la première moitié des années 1980), Társadalomtudomány, n 5, p. 35-40.

Dövényi Z. (1995). - Die strukturellen und territorialen Besonderheiten der Arbeitslosigkeit in Ungarn, In : Meusburger P., Klinger A. - dir., Vom Plan zum Markt. Eine Untersuchung am Beispiel Ungarns, Heidelberg, p. 114-129.

Dövényi Z., Kovács Z., Kok H. (1997). - A szuburbanizáció, a lokális társadalom és a hely önkormányzati politika összefüggései a budapesti agglomeráció településeiben (Suburbanisation, société locale et politique municipale dans l'agglomération de Budapest), non édité, $12 \mathrm{p}$.

Éhrlich É., Révész G., Tamás P. (1994). - Kelet-Közep-Europa : honnan hová ? (Europe centrale et orientale : bilan et perpectives), Budapest, $135 \mathrm{p}$.

Fassman H. (1992). - Phänomene der Tansformation. Ökonomische Restrukturierung und Arbeitslosigkeit in Ost-Mitteleuropa, Petermanns Geographische Mitteilungen, ${ }^{\circ}$ 136, p. 49-59.

Ferge Zs. (1986). - Fejezetek a magyar szegénypolitika történetébıl (Quelques aspects de l'histoire de la politique contre la pauvreté en Hongrie),Budapest, $156 \mathrm{p}$.

Ferge Zs. (1988). - Teljes foglalkoztatottság, foglalkoztatáspolitika, munkanélküliség (Plein emploi, politique de l'emploi, chômage), Valóság, n 6, p. 19-31.

Ferge Zs. (1996). - A rendszerváltás nyertesei és vesztesei (Les vainqueurs et les vaincus du changement de régime), Társadalmi riport, 1996, Budapest, p. 414-443.

Fóti J., Illés S. (1992). - A munkanélküliség demográfiai vonatkozásai (Les rapports démographiques du chômage), Budapest, $165 \mathrm{p}$.

Lakatos J. (1997). - Foglalkoztatottság, munkanélküliség, 1992-1996 (L'emploi et le chômage entre 1992-1996), Gazdaság és statisztika, 9. (48) 6, p. 18-31.

Tímár J. (1997). - A munkerıpiac változásai 1997 és 2002 között (Les changements du marché de l'emploi entre 1997 et 2002), Közgazdasági szemle, XLIV/11, p. 987-999.

Tolnai Gy., Dövényi Z. (1995). - A falusi munkanélküliség néhány területi és strukturális jellemvonása Magyarországon (Quelques caractéristiques territoriales et structurales du chômage rural en Hongrie), In : Kovács T., A mezıgazdaságtól a vidékfejlesztésig, Pécs, p. 449-454.

Bibliographie complémentaire :

Berényi I. (2002). - Die Auswirkungen der Privatisierung auf die Umgestaltung peripherer Räume in Ungarn, Europa Regional, n³/2002, p. 107-117.

Daroczi E. (1998). - La mobilité résidentielle en Hongrie de 1985 à 1996, Espaces, populations, sociétés, $\mathrm{n}^{\circ}$ 3, p. 381-388.

Enyedi Gy. (1996). - Regionális folyamatok Magyarországon (Différenciation régionale en Hongrie),Ember, települes, regio, Budapest, $138 \mathrm{p}$.

Fazekas K. (1997). - Válság és prosperitás a munkaeripiacon (Crises et réussites sur le marché du travail), Tér és társadalom, $\mathrm{n}^{\circ} 4$, p. 9-24. 
Des données et commentaires complémentaires peuvent être trouvés sur les sites internet de la Banque Mondiale, de l'Union Européenne et de l'Organisation de Coopération et de Développement Économique (OCDE) :

- http://europe.eu.int/comm/eurostat

- http://www.worldbank.hu

- http://www.oecd.org

\section{NOTES}

1. Correspond aux régions situées à l'ouest du Danube.

2. Conseil crée en 1949 sous l'égide de l'URSS et regroupant les états communistes de l'Europe centrale et orientale. L'acronyme anglais, COMECON, est plus utilisé que l'acronyme français.

3. Quotidien « Világgazdaság », 5 janvier 1998, p. 9.

4. Ibidem.

5. La récente décision d'implanter une très grande usine automobile du groupe Peugeot-Citroën dans la Slovaquie voisine participe à ce mouvement.

\section{RÉSUMÉS}

Ce travail s'attache à trois des conséquences socio-économiques de la transition de l'économie communiste vers l'économie de marché. Le chômage, déjà présent mais de façon rampante sous le régime communiste, s'est fortement développé au début de la transition et jusqu'en 1993, avant de décliner ensuite. L'article en étudie les différents aspects structurels et spatiaux. La pauvreté, rare en période communiste, s'est considérablement étendue, touchant de très nombreuses catégories de population : chômeurs, retraités, Tziganes. De plus, les inégalités entre une élite toujours plus riche et une masse de pauvres toujours plus importantes, se sont creusées. Enfin, le rapport migratoire villes/campagnes s'est complètement inversé, une exurbanisation inédite dans l'histoire hongroise ayant remplacé l'exode rural.

The present study deals with structural and spatial aspects of the socio-economic consequences of the change from a communist to a market economy. Unemployment, already present less obvious under the communist regime, developed strongly at the beginning of the transition and peaked in 1993 before declining. Poverty, unfamiliar during the socialist era, has expanded considerably since then, affecting many categories of people: the unemployed, pensioners, Gypsies. Furthermore, disparities between a richer elite and the larger mass of the poor increased. Migratory relationship between town and country have completely reversed, movement from urban areas, not previously experienced in Hungary, having replaced the rural exodus.

Der Aufsatz behandelt drei wichtigere soziale Folge der Wende. Die offene Arbeitslosigkeit entstand in Ungarn schon am Ende der 80er Jahre und wurde sie wegen der radikalen Umgestaltung der Wirtschaft bald massenhaft. Die Massenarbeitslosigkeit erreichte den Höhepunkt am Anfang 1993 (mehr als 700000 registrierte Arbeitslosen, die Arbeitslosenrate über 
$13 \%)$, dann verminderten sich die Werte. So war die Arbeitslosenquote am Ende 1997 etwa 10,4 \%. Im Aufsatz werden sowohl die strukturellen Besonderheiten (z.B. Qualifikations-, Alters-, Geschlechtsstruktur) als auch die räumlichen Disparitäten der Arbeitslosigkeit dargestellt. Die Armut war auch im Sozialismus nicht unbekannt, aber inzwischen ist die Anzahl der Armen mehrfach gestiegen (etwa von 1 Millionen auf 3,5 bis 4,0 Millionen). Die Einkommensunterschiede vergrösserten sich bedeutend und die Verteilung der Einkommen ist heutzutage ähnlich wie in Frankreich. Nach der Wende entstand eine in Westeuropa unbekannte Sozialstruktur, wo an der Spitze eine relativ kleine, aber sehr reiche Elite steht, die Mittelschichten immer schwächer werden und die zahlreichen Armen den Unterteil der Hierarchie bilden. Auch in der räumlicher Mobilität haben grundlegende Veränderungen im Verhältnis zum Sozialismus statt gefunden. Statt der früheren Konzentrationsprozesse wird Dekonzentration immer wichtiger. Eine eklatante Erscheinungsform dieser Wendung ist die Wohnurbanisierung, die hauptsächlich um Budapest eine grosse Bedeutung hat. In der Auswanderung aus den Städten nehmen in Ungarn nicht nur die typischen suburbanen Gruppen (Hochqualifizierte, Finanzkräfte, Mittelaltrige) teil, sondern auch die Armen, welche die wachsenden Kosten des städtischen Lebens nicht weiter bezahlen können und aus den Städte siedeln sie in die weniger attraktiven Gemeinden umsiedeln.

\section{INDEX}

Mots-clés : chômage, différences sociales, exurbanisation, pauvreté, rapports villes/campagnes Schlüsselwörter : Arbeitslosigkeit, Armut, räumliche Mobilität, soziale Differenzierung, Suburbanisierung

Keywords : out of town migration, poverty, social disparities, town/country relationship, unemployment

\section{AUTEUR}

\section{ZOLTÁN DÖVÉNYI}

Institut de Géographie de l'Académie des Sciences de Hongrie - Andrássy ut 62, 1062 Budapest HONGRIE 